\title{
A method to study the effects of chemical and biological reduction of molybdate to molybdenum blue in bacteria
}

\begin{abstract}
In this research, we modify a previously developed assay for the quantification molybdenum blue to determine whether inhibitors to molybdate reduction in bacteria inhibits cellular reduction or inhibit the chemical formation of one of the intermediate of molybdenum blue; phosphomolybdate. We manage to prove that inhibition of molybdate reduction by phosphate and arsenate is at the level of phosphomolybdate and not cellular. We also prove that mercury is a physiological inhibitor to molybdate reduction. We suggest the use of this method to assess the effect of inhibitors and activators to molybdate reduction in bacteria.
\end{abstract}

Keyword: Molybdate reduction; E. cloacae strain 48; Molybdenum blue; Inhibitors 